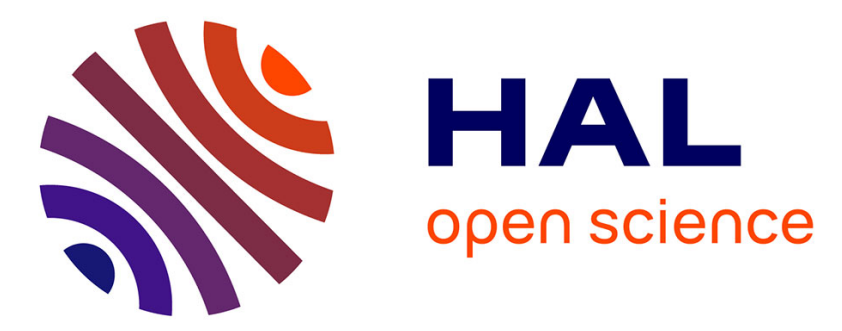

\title{
Micromechanics Contribution to Coupled Transport and Mechanical Properties of Fractured Geomaterials
}

\author{
Éric Lemarchand, Catherine A. Davy, Luc Dormieux, W. M. Chen, Frédéric
}

Skoczylas

\section{- To cite this version:}

Éric Lemarchand, Catherine A. Davy, Luc Dormieux, W. M. Chen, Frédéric Skoczylas. Micromechanics Contribution to Coupled Transport and Mechanical Properties of Fractured Geomaterials. Transport in Porous Media, 2009, 79 (3), pp.335-358. 10.1007/s11242-008-9326-5 . hal-00412574

\section{HAL Id: hal-00412574 \\ https://hal.science/hal-00412574}

Submitted on 25 Sep 2009

HAL is a multi-disciplinary open access archive for the deposit and dissemination of scientific research documents, whether they are published or not. The documents may come from teaching and research institutions in France or abroad, or from public or private research centers.
L'archive ouverte pluridisciplinaire HAL, est destinée au dépôt et à la diffusion de documents scientifiques de niveau recherche, publiés ou non, émanant des établissements d'enseignement et de recherche français ou étrangers, des laboratoires publics ou privés. 


\title{
Micromechanics Contribution to Coupled Transport and Mechanical Properties of Fractured Geomaterials
}

\author{
E. Lemarchand, C.A. Davy, L. Dormieux, W. Chen \& F. Skoczylas
}

September 25, 2009

\begin{abstract}
The present paper is devoted to the modelling of interdependent mechanical and hydraulic behaviors of geomaterials in presence of a single through-wall fracture by means of micromechanics arguments. Experimental results of fractured concrete samples show non linear evolutions for both mechanical and hydraulic behaviors with respect to confinement intensity. These non linear responses are interpreted by the progressive closure of crack-like pores defining the pore volume of the fracture interfacial domain. Disregarding tortuosity effects, we adopt a $2 \mathrm{D}$ representation for these cracks. The key role of the fracture initial porosity is also emphasized. It allows to discuss the shape of the distribution of the local apertures distribution function classically used, intercepted here in terms of the distribution of initial crack aspect ratio within the fracture domain. Application on fractured concrete samples shows the capability of the theoretical model to accurately reproduce the experimental results.
\end{abstract}

\section{Introduction}

In the context of nuclear energy use for electricity generation, radioactivity confinement is of paramount importance, both during nuclear reactor exploitation and long-term nuclear waste storage. In both life cycle phases, concrete is used for reactor structures as well as in nuclear waste packages [Davy et al., 2007]. In particular, every ten years, EDF (French Company for Electricity Supply) is compelled to subject nuclear reactor concrete vessels to air pressurizing and to check for any gas leakage. Test analysis and identification of physical mechanisms of flow through porous or even fractured medium should enable to prove if, under accidental conditions, gas leakage would remain below a given secure level or not. Nevertheless, full scale experiments are complex and their interpretation is delicate, due to the great amount of parameters involved. A more local understanding strategy, at the material scale, should therefore be considered.

Fluid flow in natural or artificially-induced fractures has been recognized for a long time as a key issue in geophysical and geomechanical engineer- 
ing ([Iwai, 1976], [Wanfang et al., 1997],[Berkowitz, 2002]). The variability of fractures geometry and its possible evolution under progressive confinement make it difficult to propose a theoretical analysis, particularly if accounting for actual couplings between mechanical and hydraulic responses. To simulate the mechanical and hydraulic responses of fractures, experimental procedures may be developed at the laboratory scale on circular cylindrical samples with a length-to-diameter ratio $(\mathrm{L} / 2 \mathrm{R})$ of about 2 at least in order to limit end effects [Davy et al., 2007]. Then, a Brazilian splitting test is performed in order to create a fracture along the sample diametral plane. Being a brittle failure test, the obtained fracture has a notable variability from one sample to another, see Figure 1. This is in accordance with the expected in situ variability.

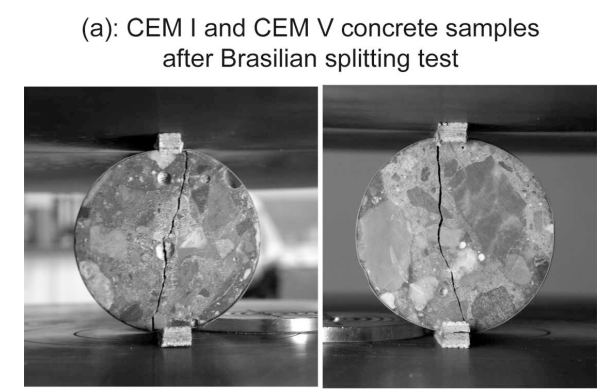

(b): Callovo-Oxfordian argillite samples after Brasilian splitting test Sample $1 \quad$ Sample 2

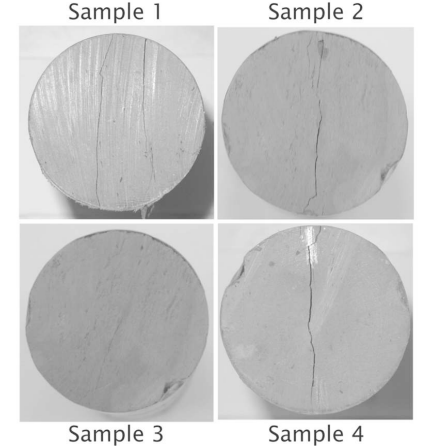

Figure 1: Photograph of various samples after Brazilian splitting test, which shows the variability in the initial fracture: (a) concrete samples; (b): CallovoOxfordian argillite.

From a phenomenological point of view, a fracture is usually interpreted as a $2 \mathrm{D}$ interface of infinitesimal thickness $\lambda$ representing the average fracture aperture (Figure 2). During macroscopic tensile or compressive loadings, denoted by $\Sigma$, the current fracture aperture is updated according to :

$$
\lambda(\Sigma)=\lambda_{0}-c_{c}(\Sigma) \quad ; \quad c_{c}\left(\Sigma_{0}\right)=0
$$




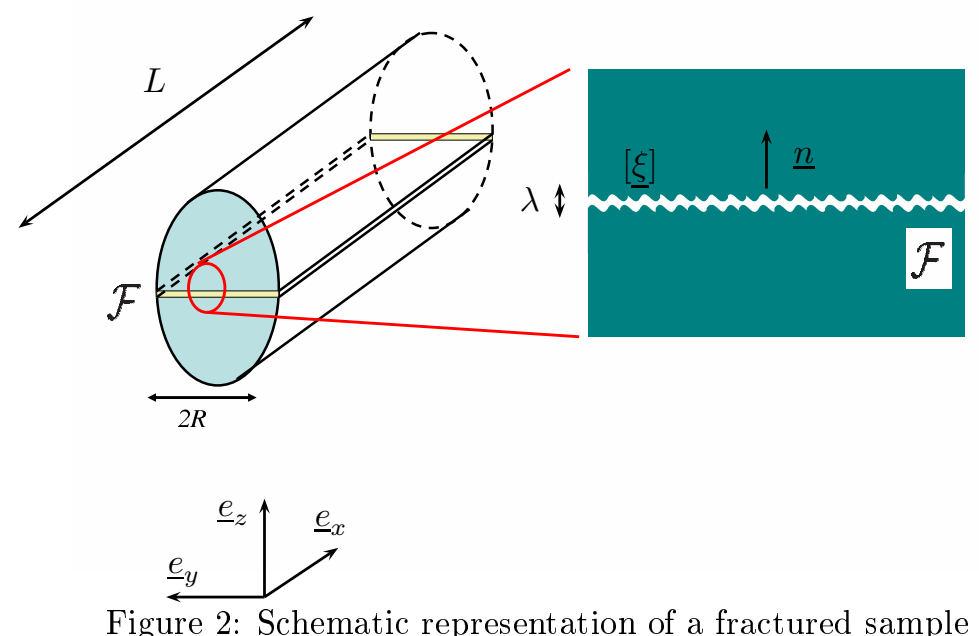

where $\lambda_{0}=\lambda\left(\Sigma=\Sigma_{0}\right)$ denotes the average fracture aperture in the reference configuration $\Sigma=\Sigma_{0}$, and $c_{c}(\Sigma)$ accounts for the evolution of the space in between the fracture faces under confinement intensity $\Sigma$. From a physical point of view, $c_{c}(\Sigma)$ represents the macroscopic mechanism of the fracture geometrical evolution, that is the fracture reclosure law for the macroscopic triaxial compressive loadings considered hereafter.

Still in this phenomenological context, determination of the hydraulic conductivity of a fracture is usually associated with the Poiseuille law [Gueguen and Palciauskas, 1992], leading to the following intrinsic permeability $\left(k^{F}=\left[m^{2}\right]\right)$ definition :

$$
\mathbf{k}^{F}=k^{F}(\mathbf{1}-\underline{n} \otimes \underline{n}) \quad ; \quad k^{F}=\frac{\lambda^{2}}{12}
$$

Recalling that the fracture volume fraction is written $\varphi^{F}=2 \lambda /(\pi R)$, and neglecting the contribution of the unfractured solid matrix, i.e. $k^{s} \ll k^{F}$ [Walters and Wong, 1999], the macroscopic permeability tensor $\mathbf{K}^{h o m}=\varphi^{F} \mathbf{k}^{F}$ reads :

$$
\mathbf{K}^{\text {hom }}=\frac{\lambda^{3}}{6 \pi R}(\mathbf{1}-\underline{n} \otimes \underline{n})
$$

In (3), the in-plane macroscopic permeability of a fractured sample appears as a cubic function of the average fracture aperture, generally referred to as the cubic law. According to the fact that this cubic law is directly associated with the parallel planes assumption, resorting to this law for real geomaterials [Witherspoon et al., 1980] seems questionable as regards the rough-walled fracture surfaces widely observed experimentally ([Tsang and Tsang, 1987], [Tsang and Tsang, 1989], 
[Wanfang et al., 1997], [Walters and Wong, 1999], [Sausse, 2002]). In particular, these surface irregularities make it difficult to properly define parameter $\lambda$ (or $\lambda_{0}$ ) that should be used in the cubic law.

For compressive loadings the progressive fracture reclosure law $c_{c}(\Sigma)$ is nonlinear in nature. This non linearity is directly related to a complex mechanism of fracture geometry evolution, which suggests a physical analysis at the scale of the interfacial geometrical irregularities. As a consequence, both mechanical and hydraulic responses of fractured geomaterials are expected to be controlled by the same physical mechanism. However, the consequences of this local mechanism may be completely different depending on the property studied, the open question being related to the existence, or not, of a correlation between the (mechanical) roughness and the (hydraulic) tortuosity. The two parallel planes assumption allowing the derivation of the hydraulic cubic law, albeit mathematically powerful, is physically not able to account for any mechanical response of the fracture. Indeed, this very specific morphology does not allow to incorporate matter in between the two parallel planes. Moreover, by nature, the cubic law is not able to discuss any physics relative to surface irregularities responsible for (mechanical) roughness and (transport) tortuosity ([Tsang, 1984],[Walters and Wong, 1999]). To overcome this limitation, many authors ([Snow, 1965],[Zimmerman and Bodvarsson, 1996] among others) have proposed to couple the cubic law with a non-linear fracture reclosure law $c_{c}(\Sigma)$. Although these models proved to be efficient, the lack of physical correlation between mechanical and hydraulic evolutions is frustrating. This is due to the fact that they are both controlled by the physics that takes place at the scale of fracture asperities [Oron and Berkowitz, 1998].

In this context, many authors have proposed improvements of the physical analysis of fractures behavior by considering that a fracture can be interpreted as a porous medium with a pore space made up of pores which morphology is likely to evolve under macroscopic confinement ([Hsieh et al., 1985], [Moreno et al., 1988], [Walsh et al., 1997], [Wanfang et al., 1997]). Among those authors, Myer [Myer, 2000] modelled a fracture by collections of cracks in order to account for the progressive fracture reclosure. In the present paper, although treated in a more general micromechanics context, our approach is similar to this morphological representation. The proposed micromechanics modelling will prove to be able to provide a physical definition of fracture aperture distribution, that has been introduced by many authors ([Neuzil and Tracy, 1981], [Tsang, 1984], [Tsang and Tsang, 1987], [Zhou and Wheater, 1995] among others.) in order to take fracture surfaces roughness into account. Besides restricting our analysis to monotonic compressive loadings, irreversibility phenomena may be disregarded and the fracture behavior is assumed non linear elastic.

\section{A Micromechanics-based analysis}

For practical purposes, non linear mechanical response of fractured geomaterials can be accounted for, in a 2D model of the fracture, by a non linear relationship 
between the fracture closure and the applied stress. The fracture closure $c_{c}(\Sigma)$ is defined as the average of the normal component of the displacement jump over the fracture, symbolized here by $\mathcal{F}$ (see Fig. 2):

$$
c_{c}=-\frac{1}{2 R L} \int_{\mathcal{F}}[\underline{\xi}] \cdot \underline{n} d S
$$

where $2 R=D$ and $L$ are respectively the sample diameter and the sample length, while $d S$ is a differential surface element.

Still, from a physical point of view, the closure of the pore space within the fracture lips is likely to be the mechanism responsible for this non linearity (see Fig. 2 ). In order to capture the pore space closure mechanism in a micromechanics framework, a 3D model of the fracture is due. At the microscopic scale, that is the one which reveals surface irregularities, the fracture of dimension $2 R \times L \times \lambda$ is interpreted as a porous medium made up of an elastic solid matrix and a fully fluid-saturated pore space (see Fig. 3) .

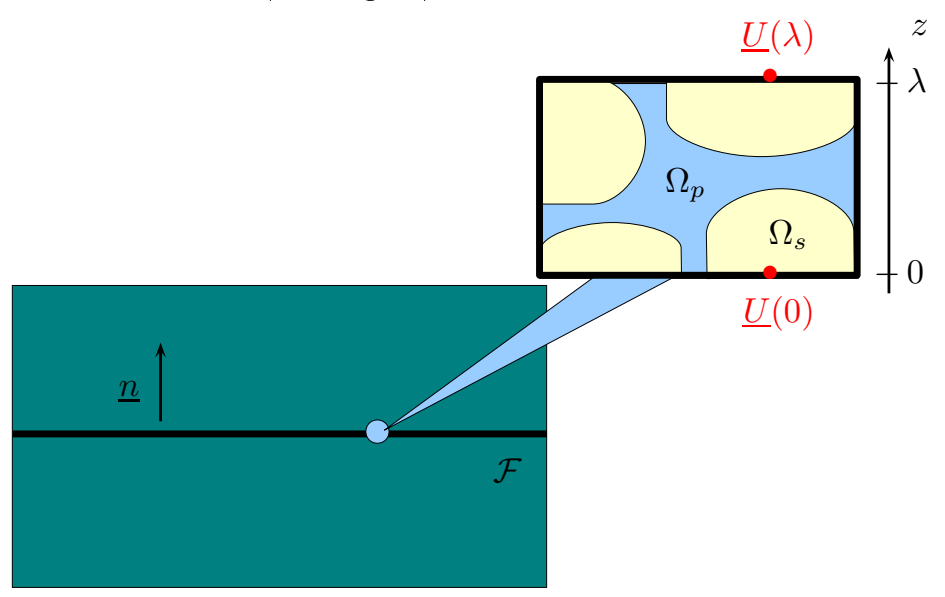

Figure 3: Macroscopic fracture interpreted as a porous medium $\left(\Omega_{F}=\Omega_{s} \bigcup \Omega_{p}\right)$

\subsection{A 3D definition of the fracture}

Let $\Omega_{s}$ (resp. $\Omega_{p}$ ) be the solid (resp. fluid) phase domain within the fracture and $V^{s}$ (resp. $V^{p}$ ) its associated volume in the current configuration. In its current configuration, the fracture domain is defined by $\Omega_{F}=\Omega_{s} \bigcup \Omega_{p}$. Its current (resp. initial) volume $V^{F}=V^{s}+V^{p}$ (resp. $\left.V_{0}^{F}=V_{0}^{s}+V_{0}^{p}\right)$, i.e. the current (resp. initial) volume is given by :

$$
V^{F}=2 R L \lambda \quad\left(V_{0}^{F}=2 R L \lambda_{0}\right)
$$

By definition, $\lambda$ is a parameter of the fracture geometry in its three dimensional description. The $2 \mathrm{D}$ displacement jump $[\xi]$ and the $3 \mathrm{D}$ displacement field $\underline{U}$ are 
related by (Fig. 3):

$$
[\underline{\xi}]=\underline{U}(\lambda)-\underline{U}(0)
$$

At the fracture level, the fracture reclosure law $c_{c}(\Sigma)$ may be directly related to the volume fraction of the pore space within the fracture domain. Let $\varphi$ denote the fracture porosity defined with respect to the current configuration of the fracture domain:

$$
\varphi=V^{p} / V^{F}
$$

We also introduce the normalized pore volume $\phi$ defined as :

$$
\phi=V^{p} / V_{0}^{F}=J \varphi
$$

where $J=\lambda / \lambda_{0}$ is the jacobian of the transformation.

Micromechanics applied to two-phase porous medium made up of an elastic solid matrix weakened by crack-like pores may prove ${ }^{1}$ that the volume strain within the solid phase is negligible when compared to the volume strain in the cracks, provided that $\varphi \ll 1$. When $\varphi=O(1)$, this result requires the solid phase incompressibility $\left(\nu_{s}=1 / 2\right)$. Let us consider an isochoric transformation of the solid matrix $\left(V^{s} \equiv V_{0}^{s}\right)$ in the fracture domain. In this case, we eventually have :

$$
\phi(\Sigma)=\frac{\lambda_{0} \phi_{0}-c_{c}(\Sigma)}{\lambda_{0}} \quad ; \quad \varphi(\Sigma)=\frac{\lambda_{0} \phi_{0}-c_{c}(\Sigma)}{\lambda_{0}-c_{c}(\Sigma)}
$$

where $\phi_{0}$ is the fracture porosity in the reference configuration.

Therefore, the evolution of the fracture pore space may be derived from the fracture reclosure law $c_{c}(\Sigma)$. In a wide range of experimental results, the evolution law $c_{c}(\Sigma)$ follows a trend that may be fitted by the non-linear law :

$$
\forall \Sigma \geq \Sigma_{0} \quad, \quad c_{c}=c_{c}^{\infty}\left(1-\exp \left(\frac{\Sigma_{0}-\Sigma}{\Sigma_{\infty}}\right)\right)
$$

In practice, the slope at the origin $\left(\Sigma \rightarrow \Sigma_{0}\right)$ and the asymptote for infinite values of $\Sigma$ allow the determination of both parameters $c_{c}^{\infty}$ and $\Sigma_{\infty}$.

From (10) analytical definitions of fracture porosity $\varphi$ and normalized pore volume $\phi$ are derived. Assuming a complete reclosure of the fracture pore space for infinite value of the macroscopic confining pressure $\Sigma,(9)$ and (10) yield:

$$
\lim _{\Sigma \rightarrow \infty} \varphi=0 \quad \Leftrightarrow \quad \phi_{0}=\frac{c_{c}^{\infty}}{\lambda_{0}}
$$

so that (9) may be rewritten as :

$$
\left\{\begin{array}{l}
\phi(\Sigma)=\phi_{0} \exp \left(\left(\Sigma_{0}-\Sigma\right) / \Sigma_{\infty}\right) \\
\varphi(\Sigma)=\frac{\phi_{0} \exp \left(\left(\Sigma_{0}-\Sigma\right) / \Sigma_{\infty}\right)}{1-\phi_{0}\left(1-\exp \left(\left(\Sigma_{0}-\Sigma\right) / \Sigma_{\infty}\right)\right)}
\end{array}\right.
$$

\footnotetext{
${ }^{1}$ by resorting to micromechanics analysis (Mori-Tanaka's scheme for instance)
} 
It is important to note that experimental results allow to determine the evolution of the volume fraction of the fracture pore space with respect to the compressive loading intensity. Since we are dealing with a given displacement-stress relationship rather than a strain-stress relationship, one information is still missing for the macroscopic behavior description. Indeed $\phi_{0}$ (or equivalently $\lambda_{0}$ ) appears in (12) as an unknown parameter that still has to be determined, or at least estimated. In particular, its fictitious nature does not permit a reliable measurement of $\phi_{0}$.

Moreover, it should be emphasized that (11) introduces a strong link between material effects, through $\phi_{0}$, and structural effects, through $\lambda_{0}$. From (11), those effects cannot be considered separately. For any value of $\phi_{0}$ corresponds a unique value of $\lambda_{0}$ consistent with the fracture reclosure mechanism experimentally identified $\left(c_{c}^{\infty}\right)$. This competition between material and structural effects is expected to influence both mechanical and hydraulic behaviors.

\section{$2.2 \quad$ A micromechanics model}

Following the morphological representation of fracture aperture distribution proposed by Myer ([Myer, 2000], [Jaeger et al., 2007]), we assume from now on that the fracture pore space is made up of a distribution of crack-like pores having different initial aspect ratios. As a consequence, fracture porosity is completely determined by the total cracks volume $V^{p}=V^{c}$. This morphological assumption allows to address the progressive fracture reclosure along the applied confining pressure, on the basis of the progressive reclosure of local cracks, from the smaller to the higher aspect ratios.

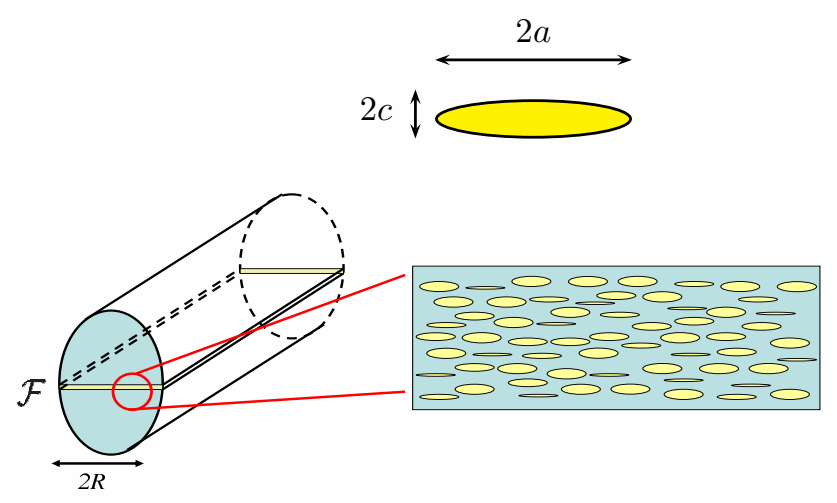

Figure 4: The fracture modelled as a distribution of discrete parallel cylinders

In view of applying Eshelby's work [Eshelby, 1957], a crack is here modelled as a cylindrical inclusion [Horii and Nemat-Nasser, 1983], of length $L$, with an 
elliptical section embedded in a solid matrix (see Fig. 4). These inclusions are introduced so as to account for the anisotropic situation where parallel cracks are uniformly distributed. The unit normal vector $\underline{n}=\underline{e}_{z}$ is the same for all cracks. Crack radius is denoted by $a$ and crack half-opening by $c$. Crack aspect ratio is denoted by $X=c / a \ll 1$. For the sake of simplicity, cracks are assumed to have the same radius $a$, so that a crack family is completely defined by its initial aspect ratio.

Let $\nu\left(X_{0}\right)$ be the distribution function of crack aspect ratios in the initial state. By definition, $\nu\left(X_{0}\right) d X_{0}$ stands for the number of cracks per unit length (i.e. diameter $2 R$ in the fracture plane) which initial aspect ratio lies in between $X_{0}$ and $X_{0}+d X_{0}$. According to (8) where $V^{p}=V^{c}$, the incremental pore volume fraction is then given by :

$$
d \varphi=\frac{X}{J} \epsilon\left(X_{0}\right) d X_{0} \quad ; \quad \epsilon\left(X_{0}\right)=\frac{\pi a^{2}}{\lambda_{0}} \nu\left(X_{0}\right)
$$

where $\epsilon\left(X_{0}\right)$ is a $2 \mathrm{D}$ crack density parameter, which is similar to the crack density parameter introduced by [Budiansky and O'Connell, 1976]. (13) then allows us to give a micromechanics definition for the fracture volume fraction introduced in (7):

$$
\varphi(\Sigma)=\frac{1}{J(\Sigma)} \int_{\mathcal{O}(\Sigma)} X \epsilon\left(X_{0}\right) d X_{0}
$$

where $\mathcal{O}(\Sigma)$ formally represents the set of open cracks for a macroscopic stress intensity $\Sigma$, i.e. integrations are made over the initial aspect ratio of cracks families still open for confining pressure intensity $\Sigma$.

\subsubsection{Local crack closure law}

According to the fact that cracks are likely to undergo non-infinitesimal deformations under macroscopic compressive stresses, we have to resort to a rate-based formulation of the constitutive equations. In this framework, it is then possible to derive an estimate for the relation existing between the local strain rate $\boldsymbol{d}$ prevailing within a crack and the macroscopic applied stress rate $\dot{\boldsymbol{\Sigma}}$. Let us consider a representative element volume of the fracture made up of an elastic solid matrix (volume fraction $f_{s}=1-\varphi$, elastic stiffness tensor $\mathbb{C}^{s}$ ) and identical oriented cracks (total volume fraction $f_{c}=\varphi$, elastic stiffness tensor $\mathbb{C}^{c}$ ) submitted to a macroscopic strain rate $\mathbf{D}$. In continuum micromechanics, use of an elastic tensor $\mathbb{C}^{c}$ allows accounting for different behavior of the cracks. For instance [Dormieux et al., 2006], $\mathbb{C}^{c}=0$ for open cracks, while $\mathbb{C}^{c} \neq 0$ may represent closed cracks. In this latter case, $\mathbb{C}^{c}$ is defined in order to account for the behavior (frictionless or not) of the cracks.

The linearity of the problem addressed in rate formulation allows us to relate linearly the microscopic and macroscopic strain rates according to $^{2}$ :

$$
\boldsymbol{d}^{\alpha}=\overline{\mathbb{A}}^{\alpha}: \boldsymbol{D}
$$

${ }^{2} \bar{y}^{\beta}$ denotes the average of $y$ over domain $\left.\Omega_{\beta}(\beta=s, c, F)\right)$ 
where $\overline{\mathbb{A}}^{\alpha}$ denotes the average concentration tensor overdomain $\Omega_{\alpha}(\alpha=s$ (resp. c) for the solid phase (resp. cracks)). Estimates for these concentration tensors are derived from micromechanics homogenization schemes ([Mura, 1987],[Dormieux et al., 2006]). In the framework of the Mori-Tanaka scheme, estimates for localization tensors read :

$$
\left\{\begin{array}{l}
\overline{\mathbb{A}}^{c}=\left(\mathbb{I}+\mathbb{P}_{c}^{s}:\left(\mathbb{C}^{c}-\mathbb{C}^{s}\right)\right)^{-1}: \tilde{\mathbb{A}} \\
\overline{\mathbb{A}}^{s}=\tilde{\mathbb{A}}
\end{array}\right.
$$

where $\mathbb{I}$ and $\mathbb{P}_{c}^{s}$ are the fourth order identity tensor and Hill's tensor associated with a cylindrical inclusion of elliptical cross-section embedded in the solid matrix of stiffness tensor $\mathbb{C}^{s}$ respectively, while

$$
\tilde{\mathbb{A}}^{-1}=f_{s} \mathbb{I}+\frac{1}{J} \int_{\mathcal{O}(\Sigma)} X\left(\mathbb{I}+\mathbb{P}_{c}^{s}:\left(\mathbb{C}^{c}-\mathbb{C}^{s}\right)\right)^{-1} \epsilon\left(X_{0}\right) d X_{0}
$$

is introduced so as to satisfy the strain rate average rule $\overline{\boldsymbol{d}}^{F}=\boldsymbol{D}$. Considering open cracks, we have $\mathbb{C}^{c}=0$ so that the strain rate in a crack may be estimated as [Dormieux et al., 2006] :

$$
\boldsymbol{d}^{c}=\left(\mathbb{I}-\mathbb{S}_{c}^{s}\right)^{-1}: \tilde{\mathbb{A}}_{\mid \mathbb{C}^{c}=0}: \boldsymbol{D}
$$

where $\mathbb{S}_{c}^{s}=\mathbb{P}_{c}^{s}: \mathbb{C}^{s}$ is the Eshelby tensor.

Defining the rate of the macroscopic stress as the average over the r.e.v. of the local stress rates, say $\dot{\boldsymbol{\Sigma}}=\overline{\boldsymbol{\sigma}}^{F}$, it is found that the fracture behavior itself takes an hypoelastic form $\left(\mathbb{S}_{t}^{\text {hom }}=\left(\mathbb{C}_{t}^{\text {hom }}\right)^{-1}\right)$ :

$$
\dot{\boldsymbol{\Sigma}}=\mathbb{C}_{t}^{\text {hom }}: \boldsymbol{D} \quad \text { or } \quad \boldsymbol{D}=\mathbb{S}_{t}^{\text {hom }}: \dot{\boldsymbol{\Sigma}}
$$

where homogenized stiffness tensor $\mathbb{C}_{t}^{h o m}=\overline{\mathbb{C}: \mathbb{A}}^{F}=(1-\varphi) \mathbb{C}^{s}: \overline{\mathbb{A}}^{s}$. For hydrostatic loading conditions, (19) allows us to relate the fracture strain rate $\boldsymbol{D}$ to the rate of the macroscopic confining pressure $\dot{\boldsymbol{\Sigma}}=-\dot{\Sigma} \mathbf{1}(\Sigma>0$ for compression) as :

$$
\boldsymbol{D}=-\dot{\Sigma} \mathbb{S}_{t}^{\text {hom }}: \mathbf{1}
$$

Combining (16), (18), (19) and (20), the crack closure law can be derived from the relation $\underline{n} \otimes \underline{n}: \boldsymbol{d}^{c}=d_{n n}^{c}=\dot{c} / c$ [Deudé et al., 2002] :

$$
\dot{X}=\frac{\dot{c}}{a}=X d_{n n}^{c}=-\alpha_{n}^{s} \frac{\dot{\Sigma}}{1-\varphi(\Sigma)}
$$

where possible crack propagation is disregarded $(\dot{a}=0)$ and where coefficient $\alpha_{n}^{s}$ is given by :

$$
\alpha_{n}^{s}=\underline{n} \otimes \underline{n}: X\left(\mathbb{I}-\mathbb{S}_{c}^{s}\right)^{-1}: \mathbb{S}^{s}: 1
$$

Interestingly, one can prove (see for instance [Dormieux et al., 2006]) that $\mathbb{T}^{s}=$ $X\left(\mathbb{I}-\mathbb{S}_{c}^{s}\right)^{-1}$ is a tensorial function independent of crack aspect ratio provided that the latter is small enough $X \ll 1$ (which is true for cracks by definition) and function of the solid matrix Poisson ratio $\nu_{s}$ only. For parallel cylindrical 
inclusions of elliptical section with aspect ratios $X \ll 1$ (unit normal $\underline{n}=\underline{e}_{z}$ ) in an isotropic solid matrix, one gets :

$$
\alpha_{n}^{s}=2\left(1-\nu_{s}^{2}\right) / E_{s} \quad\left(=\left(1-\nu_{s}\right) / \mu_{s}\right)
$$

Interestingly enough, (23) proves that the local mechanical response (crack closure law) is mainly controlled by the solid matrix Young modulus. Therefore, solid matrix incompressibility assumption $\left(\nu_{s}=1 / 2\right)$ may be used without modifying the result (see section 2.1).

Time integration of (21) yields the evolution law of crack aspect ratio with respect to the applied loading ${ }^{3}$ :

$$
X=\left\langle X_{0}-X_{0}^{c \ell}(\Sigma)\right\rangle_{+} \quad \text { with } \quad X_{0}^{c \ell}(\Sigma)=\alpha_{n}^{s} \int_{\Sigma_{0}}^{\Sigma} \frac{d s}{1-\varphi(s)}
$$

where $X_{0}=X\left(\Sigma_{0}\right)$ is the crack aspect ratio in the reference configuration $\Sigma=\Sigma_{0} . X_{0}^{c \ell}(\Sigma)$ represents the initial aspect ratio of the cracks which close when the confining pressure reaches $\Sigma$. In other words, for a given value of $\Sigma$, cracks which are still open are those which initial aspect ratios greater than the threshold value $X_{0}^{c \ell}(\Sigma)$. Taking advantage of $(12), X_{0}^{c \ell}(\Sigma)$ may also be written :

$$
X_{0}^{c \ell}(\Sigma)=\alpha_{n}^{s}\left(\left(\Sigma-\Sigma_{0}\right)-\eta(\Sigma)\right) \quad, \forall \Sigma \geq \Sigma_{0}
$$

where $\eta(\Sigma)=\Sigma_{\infty}\left(\phi(\Sigma)-\phi_{0}\right) /\left(1-\phi_{0}\right)$. Therefore, at confining pressure $\Sigma$, the set of open cracks $\mathcal{O}(\Sigma)$ introduced in (14) gathers cracks with initial aspect ratio satisfying the condition $X_{0}>X_{0}^{c \ell}(\Sigma)$, where $X_{0}^{c \ell}(\Sigma)$ is given in (25). As a consequence, the fracture porosity now reads :

$$
\varphi(\Sigma)=\frac{1}{J(\Sigma)} \int_{X_{0}^{c \ell}(\Sigma)}^{\infty}\left(X_{0}-X_{0}^{c \ell}(\Sigma)\right) \epsilon\left(X_{0}\right) d X_{0}
$$

Conversely, the closure of a given crack family $(X=0)$ occurs as soon as the applied stress reaches $\Sigma^{c \ell}\left(X_{0}\right)$ satisfying $X_{0}^{c \ell}\left(\Sigma=\Sigma^{c \ell}\left(X_{0}\right)\right)=X_{0}$. According to $(25), \Sigma^{c \ell}\left(X_{0}\right)$ reads :

$$
\Sigma^{c \ell}\left(X_{0}\right)=\Sigma_{0}-\Sigma_{\infty}\left(h\left(X_{0}\right)-W\left(\frac{\phi_{0}}{1-\phi_{0}} e^{h\left(X_{0}\right)}\right)\right)
$$

where $h\left(X_{0}\right)=\frac{\phi_{0}}{1-\phi_{0}}-\frac{X_{0}}{\alpha_{n}^{s} \Sigma_{\infty}}$, while the Lambert function $W(x)$ satisfies :

$$
W(x) \times \exp (W(x))=x
$$

Taking advantage of (12), the crack density parameter $\epsilon\left(X_{0}\right)$ may easily be derived from a double derivative ([Morlier, 1971], [Jaeger et al., 2007], [Deudé et al., 2002]) of (26) with respect to $\Sigma$ as :

$$
\begin{aligned}
\epsilon\left(X_{0}\right)=\frac{\phi_{0}}{\Sigma_{\infty}^{2}} \exp \left(\left(\Sigma_{0}-\Sigma^{c \ell}\left(X_{0}\right)\right) / \Sigma_{\infty}\right) \\
\times\left[\left(\frac{d X_{0}^{c \ell}}{d \Sigma}+\Sigma_{\infty} \frac{d^{2} X_{0}^{c \ell}}{d \Sigma^{2}}\right) /\left(\frac{d X_{0}^{c \ell}}{d \Sigma}\right)^{3}\right]_{\mid \Sigma=\Sigma^{c \ell}\left(X_{0}\right)}
\end{aligned}
$$

\footnotetext{
${ }^{3}\langle\cdot\rangle_{+}$stands for the "positive part of"
} 
Although directly linkted to the fracture reclosure law (12), (29) proves that the initial crack aspect ratio distribution only requires an estimate for the crack aspect ratio threshold $X_{0}^{c \ell}(\Sigma)$.

Use of (25) together with (27) in (29) yield the crack density parameter :

$$
\epsilon\left(X_{0}\right)=\frac{1-\phi_{0}}{\left(\alpha_{n}^{s} \Sigma_{\infty}\right)^{2}} \varphi\left(\Sigma^{c \ell}\left(X_{0}\right)\right)\left[1-\varphi\left(\Sigma^{c \ell}\left(X_{0}\right)\right)\right]^{2}
$$

Remarkably, the Mori Tanaka effective media theory provides a micromechanics interpretation of the mechanical response of the fracture using the crack reclosure law (25) and the initial crack aspect ratio distribution (30). In particular, (30) proves that $\epsilon\left(X_{0}\right)$ depends upon three parameters: $\alpha_{n}^{s}, \Sigma_{\infty}$ and $\phi_{0}$. Assuming that the solid matrix elastic coefficients $\left(E_{s}, \nu_{s}=1 / 2\right)$ are given quantities, $\alpha_{n}^{s}$ is estimated by (23). The shape of $\epsilon\left(X_{0}\right)$ is then controlled by $\Sigma_{\infty}$ and $\phi_{0}$. Interestingly, as in [Oron and Berkowitz, 1998] the derived initial crack aspect ratio distribution shows that contact points having zero aperture in fractures are naturally managed by our micromechanics modelling whatever the value of $\phi_{0} \neq 0$. For convenience, we introduce from now on the normalized initial crack aspect ratio distribution $\hat{\epsilon}\left(X_{0}\right)=\epsilon\left(X_{0}\right) / \epsilon(0)$.

A simple derivation of (30) proves that the normalized crack density parameter $\hat{\epsilon}\left(X_{0}\right)$ may exhibit a maximum value $\tilde{\epsilon}=\hat{\epsilon}\left(X_{0}=\tilde{X}_{0}\right)$ at $\varphi=1 / 3$ :

$$
\tilde{\epsilon}=\frac{4}{27} \frac{1}{\phi_{0}\left(1-\phi_{0}\right)^{2}}
$$

Introducing $u=2 \phi_{0} /\left(1-\phi_{0}\right)$, the initial crack aspect ratio $\tilde{X}_{0}$ associated with $\tilde{\epsilon}$ also reads :

$$
\tilde{X}_{0}=\left(\alpha_{n}^{s} \Sigma_{\infty}\right) / 2 \times(u-1+2 \ln (u))
$$

Due to the (required) positivity of $\tilde{X}_{0}$, the existence of $\tilde{\epsilon}$ is only ensured for $1 / 3 \leq \phi_{0} \leq 1$. When $\phi_{0} \leq 1 / 3$, the maximum value is $\tilde{\epsilon}=1$.

\subsubsection{Effect of $\phi_{0}$ on the initial crack aspect ratio distribution}

As previously mentionned, $\phi_{0}$ controls the intensity (31) and the position (32) of the peak of the initial aspect ratio distribution. In particular, depending upon $\phi_{0}$, function $\hat{\epsilon}\left(X_{0}\right)$ may take different shapes as shown in Fig. 5 for $\Sigma_{\infty}=1$ $\mathrm{MPa}$ and $\alpha_{n}^{s}=5 \cdot 10^{-5} \mathrm{MPa}^{-1}$.

- if $\phi_{0}<1 / 3$, see Fig. 5(c), the micromechanics model suggests a decreasing function of an exponential type. As $\phi_{0} \rightarrow 0$, this result becomes increasingly equivalent to the one obtained by assuming non-interactive crack-like pores as $\phi_{0} \rightarrow 0$ (see appendix).

- if $1>\phi_{0}>1 / 3$, the micromechanics model produces a truncated-gaussian type function, see Fig. 5(b). The latter has been measured experimentally ([Billaux et al., 1984], [Gentier, 1986], [Walters and Wong, 1999]) and commonly fitted by gamma or log-normal functions in theoretical or numerical approaches for defining local apertures distribution of real fractures ([Neuzil and Tracy, 1981], [Tsang and Tsang, 1987], [Renshaw, 1995]). 
- if $\phi_{0} \rightarrow 1$, a skewed, almost gaussian, function is obtained, see Fig. 5(a). This type of function has also been used by several authors as the local apertures distribution associated with fractures ([Tsang, 1984],[Pyrak-Nolte and Morris, 2000]). It has also been obtained following numerical simulations ([Tsang and Tsang, 1989], [Unger and Mase, 1993], [Oron and Berkowitz, 1998]).

\subsubsection{Characterization of $\phi_{0}$}

Interestingly, whatever the value of $\phi_{0}$, when the normalized crack density parameter $\hat{\epsilon}\left(X_{0}\right)$ is an input of the problem, the latter may provide a characterization of $\phi_{0}$, provided that $\Sigma_{\infty}$ and $\alpha_{n}^{s}$ are given quantities.

- when $1 / 3 \leq \phi_{0} \leq 1$, we make use of the existence of a maximum of $\hat{\epsilon}\left(X_{0}\right)$. It is then straightforward to determine $\phi_{0}$ by using (32) since $\tilde{X}_{0}$ is here an input of the problem.

- when $\phi_{0} \leq 1 / 3$, we make use of the slope at the origin of $\hat{\epsilon}\left(X_{0}\right)$, denoted by $\bar{\eta}$. According to (30), the latter takes the form:

$$
\bar{\eta}=\frac{d \hat{\epsilon}}{\left.d X_{0}\right|_{0}=0}=\frac{1}{\alpha_{n}^{s} \Sigma_{\infty}} \frac{(2 W(g(u))-1)}{(W(g(u))+1)^{2}}
$$

where $g(u)=\frac{u}{2} \exp \left(\frac{u}{2}\right)$ with $u=2 \phi_{0} /\left(1-\phi_{0}\right)$.

More generally, determination of $\phi_{0}$ from a purely mechanical analysis requires three informations:

- the solid matrix (assumed isotropic elastic) behavior providing $\alpha_{n}^{s}$,

- the experimental fracture reclosure law providing $\Sigma_{\infty}$ according to the analytical approximation introduced in (10),

- the initial crack aspect ratio distribution $\epsilon\left(X_{0}\right)$, similar to local fracture apertures distribution, provided by experimental characterization techniques.

\subsubsection{Effect of $\Sigma_{\infty}$ on the nature of a fracture}

In [Tsang, 1984], an interesting qualitative classification of geometrical fracture states is given, depending upon the shape of the fracture aperture density function. Sharply peaked aperture distributions, whether gaussian or skewed (see Fig. 5 a) and b)) are characteristic of very well matched fractures, while ill-mated fractures are rather associated with flat and broad apertures distribution ([Tsang, 1984],[Berkowitz, 2002]).

Our micromechanics modelling has been developped in a sufficiently large framework to manage any type of fracture. Assuming that $\alpha_{n}^{s}$ is a given quantity, the initial crack aspect ratio distribution depends upon $\phi_{0}$ and $\Sigma_{\infty}$. As previously presented, $\phi_{0}$ provides the trend of the initial crack aspect ratio distribution. 


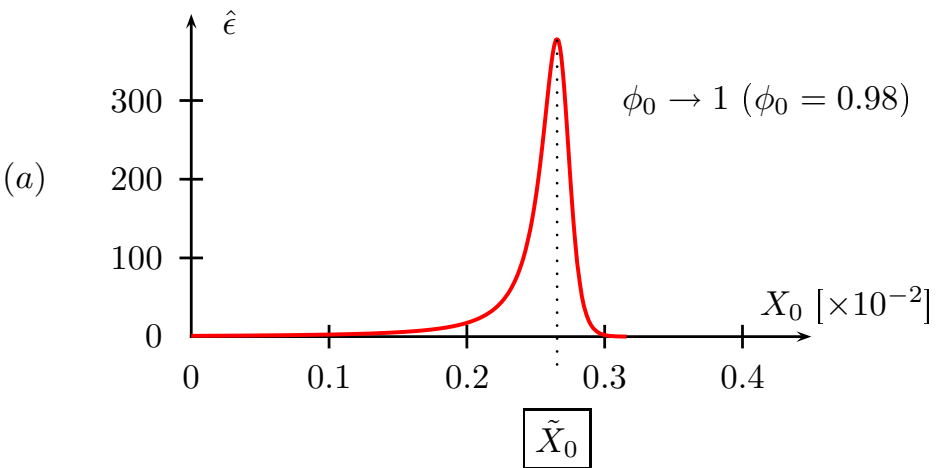

(b)

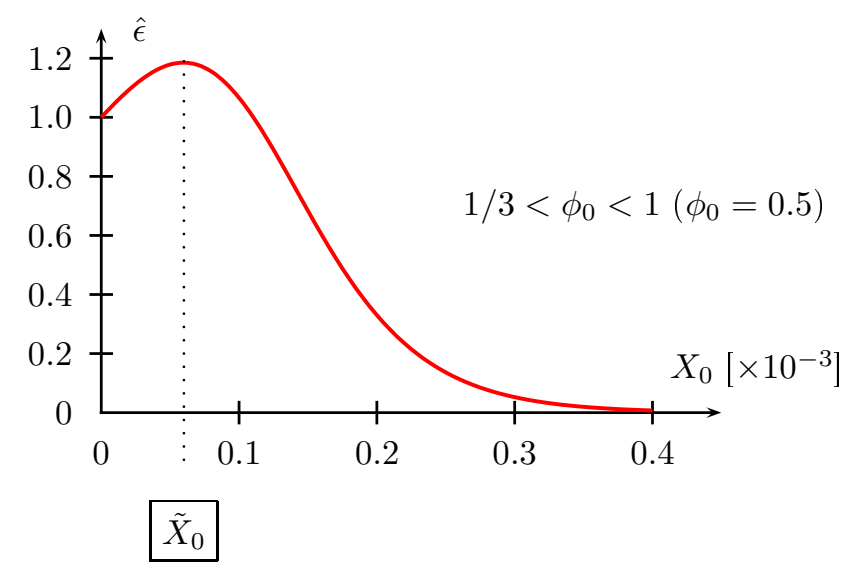

$(c)$

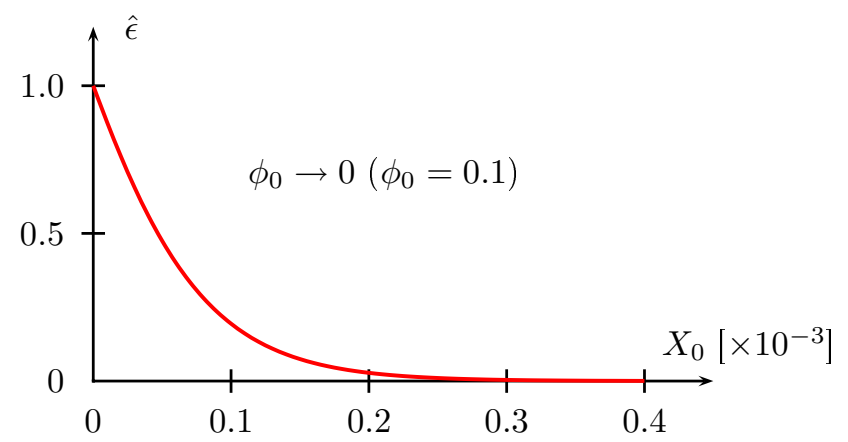

Figure 5: Normalized initial crack asp£8t ratio distribution as a function of $\phi_{0}$ $\left(\alpha_{n}^{s}=5 e^{-5} \mathrm{MPa}^{-1}, \Sigma_{\infty}=1 . \mathrm{MPa}\right)$ 
However, there is no way to associate the value of the initial fracture pore volume $\phi_{0}$ to the well-matched or ill-mated state of the fracture. By contrast, depending on parameter $\Sigma_{\infty}$, (30) may exhibit a sharply peaked shape $\left(\Sigma_{\infty}\right.$ small enough) or a flat and broad shape ( $\Sigma_{\infty}$ sufficiently high). This is illustrated in figure 6 where we used $\Sigma_{\infty}=1 \mathrm{MPa}$ (resp. 5MPa) for the peaked (resp. flat) distribution. Obviously, the proposed micromechanics approach is able to retrieve classical fracture apertures distribution shape [Tsang, 1984]. In addition, this model allows us to give a qualitative and quantitative explanation to the well-matched or ill-mated definition of a fracture. Let us consider that the macroscopic fracture reclosure occurs for a confining pressure $\Sigma=\Sigma^{*}=7 \mathrm{MPa}$. Let us also consider two different values for $\Sigma_{\infty}=1 \mathrm{MPa}, 5 \mathrm{MPa}$ respectively. According to (25) with $\phi_{0}=0.5$ and $\alpha_{n}^{s}=5 e-5 \mathrm{MPa}^{-1}$, the initial aspect ratio threshold satisfies :

$$
X_{0}^{c \ell}\left(\Sigma^{*}\right)= \begin{cases}4 e^{-4} & \text { for } \Sigma_{\infty}=1 \mathrm{MPa} \\ 5.38 e^{-4} & \text { for } \Sigma_{\infty}=5 \mathrm{MPa}\end{cases}
$$

Therefore, for $\Sigma_{\infty}=1 \mathrm{MPa}$ (resp. $5 \mathrm{MPa}$ ) the fracture reclosure at confining pressure $\Sigma^{*}=7 \mathrm{MPa}$ is associated with the reclosure of the cracks with an initial aspect ratio $X_{0} \leq 4 e^{-4}$ (resp. $X_{0} \leq 5.38 e^{-4}$ ). From figure $6, \Sigma_{\infty}=1$ MPa yields a complete reclosure of the local crack-like pores, consistent with a well-matched definition of the fracture. By contrast, $\Sigma_{\infty}=5 \mathrm{MPa}$ yields a partial reclosure of the local crack-like pores, which can be related to an ill-mated fracture. This result can also be interpreted through the definition of $\tilde{X}_{0}$ given in (32). When $\Sigma_{\infty}$ increases, the maximum value $\tilde{\epsilon}$ is associated with a higher value of $\tilde{X}_{0}$. Hence, a higher value of the confining pressure is required for the complete closure of the local crack-like pores.

When $\phi_{0}<1 / 3$, the same conclusion is obtained. According to (33), for increasing values of $\Sigma_{\infty}$, the initial crack aspect ratio distribution tends toward the line $\hat{\epsilon}=1$.

Whithin our analysis, determination of both crack reclosure law (25) and initial crack aspect ratio distribution (30) has to be understood as the key of the micromechanics interpretation of the fracture reclosure mechanism. In particular, the proposed analysis proves that, whatever $\phi_{0}$, we are able to build an initial crack aspect ratio distribution $\epsilon\left(X_{0}\right)$ that is compatible with the observed macroscopic behavior (fracture reclosure). This result is a direct consequence of the relation between $\phi_{0}$ (material effects) and $\lambda_{0}$ (structural effects) expressed in (11). Whenever the material $\left(\phi_{0}\right)$ is modified, the initial fracture thickness $\left(\lambda_{0}\right)$ itself is modified in such a way that they are still associated with the same macroscopic behavior.

Hence, the mechanical analysis does not allow the determination of $\phi_{0}$, excepted the situation where all needed informations are known as mentionned in section 2.2.3. In order to overcome this indetermination on $\phi_{0}$, we may take advantage of the hydraulic behavior of fractured geomaterials. This point is adressed in the next section, where permeability evolution of fractured geomaterials are estimated in the context of micromechanics arguments, still based on the MoriTanaka effective media theory. 


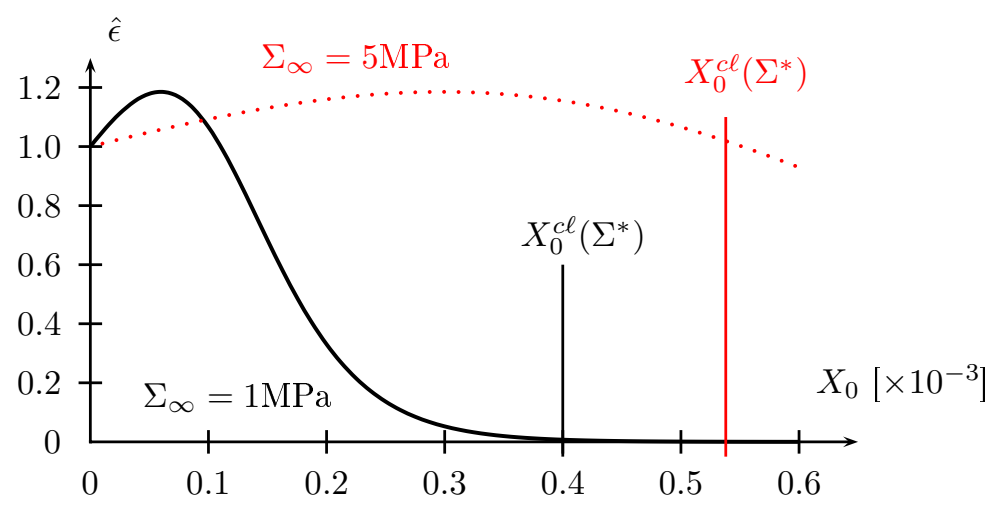

Figure 6: Effect of $\Sigma_{\infty}$ on the fracture definition $\left(\alpha_{n}^{s}=5 e^{-5} \mathrm{MPa}^{-1}, \phi_{0}=0.5\right)$

\section{3 fracture permeability estimates}

We take here advantage of classical rock joints conduction laws as proposed in Rock Hydraulics ([Gueguen and Palciauskas, 1992], [Dormieux and Lemarchand, 2001]). The idea is as simple as considering a crack as a system of two parallel planes, of width $2 c$. In between these two planes, the fluid obeys a Poiseuille conduction law that linearly relates the filtration velocity and the pressure gradient. It is therefore possible to define a fictitious porous medium of permeability $\boldsymbol{k}_{c}$, that is equivalent to the real crack as regards the linear relationship between filtration velocity and pressure gradient. Recalling that $\underline{e}_{x}$ and $\underline{e}_{y}$ are the two orthonormal vectors in the crack plane, and $\underline{n}=\underline{e}_{z}$ the outward unit normal to this plane, we may write :

$$
\boldsymbol{k}_{c}=\frac{c^{2}}{3}\left(\underline{e}_{x} \otimes \underline{e}_{x}+\underline{e}_{y} \otimes \underline{e}_{y}\right)+k_{s} \underline{n} \otimes \underline{n}
$$

where arbitrarily defined parameter $k_{s}$ may be chosen as the (isotropic) permeability of the uncracked solid matrix $\boldsymbol{k}_{s}=k_{s} \mathbf{1}$ [Dormieux and Kondo, 2004]. It is readily seen that the macroscopic permeability tensor $\boldsymbol{K}^{\text {hom }}$ is strongly affected by the presence of crack through the fact that it locally enhances (if $\left.c^{2} \gg k_{s}\right)$ the fluid motion in the $\left(\underline{e}_{x}, \underline{e}_{y}\right)$ plan. By contrast, its effect on the fluid flow in the normal direction $\underline{n}$ is negligible. From a physical point of view, replacing the fluid domain in a crack by a fictitious porous medium, of permeability $\boldsymbol{k}_{c}$, is consistent with the fact that the linearity of the local conduction law is preserved. On a mathematical point of view, this substitution is very powerful. Indeed, it replaces the difficult task of the homogenization process of a Stokes problem over a representative element volume (r.e.v.) by the one of an heterogeneous darcean medium with heterogeneous permeability $\boldsymbol{k}(\underline{z})$. Considering again a continuous distribution of initial crack aspect ratios, each crack 
family $\left(\mathcal{F}^{j}\right)$ accounts for one distinct phase as regards the heterogeneous description of Darcy's law within the r.e.v..

With the assumption that $k_{s} \approx 0$ [Walters and Wong, 1999], micromechanics tools then allow to derive fracture permeability as the average over the r.e.v., denoted by $\langle\cdot\rangle$, according to :

$$
\mathbf{k}^{F}=\langle\mathbf{k} \cdot \mathbf{A}\rangle=\frac{1}{J} \int_{X_{0}^{c \ell}(\Sigma)}^{\infty} \mathbf{k}_{c} \cdot \mathbf{A}_{c} X \epsilon\left(X_{0}\right) d X_{0}
$$

As the second order concentration tensor reads ${ }^{4} \mathbf{A}_{c}=\underline{e}_{x} \otimes \underline{e}_{x}$ with the morphology assumption made in section 2.2, tortuosity effects cannot be accounted for in the present analysis [Dormieux and Lemarchand, 2000]. Therefore, (36) reduces to :

$$
\mathbf{k}^{F}=\frac{1}{J} \int_{X_{0}^{c \ell}(\Sigma)}^{\infty} \frac{c^{2}}{3} X \epsilon\left(X_{0}\right) d X_{0} \underline{e}_{x} \otimes \underline{e}_{x}
$$

At the sample scale, as we chose a specific two layers composite morphology (see Fig. 3), the macroscopic permeability tensor is derived by a simple mixture law :

$$
\mathbf{K}^{\text {hom }}=\varphi^{F} \mathbf{k}^{F} \quad ; \quad \varphi^{F}=2 \lambda /(\pi R)
$$

Combining Eqns (37) and (38) finally yields a longitudinal macroscopic permeability coefficient estimated by :

$$
K_{x x}^{h o m}=\frac{2 a^{2} \lambda_{0}}{3 \pi R} \int_{X_{0}^{c \ell}(\Sigma)}^{\infty} X^{3} \epsilon\left(X_{0}\right) d X_{0}
$$

This is similar to the macroscopic permeability proposed in [Tsang and Witherspoon, 1983]. Hence, resorting to mixture law estimates yields a macroscopic longitudinal permeability coefficient defined as a cubic function of crack aspect ratio, classically referred to as a "local cubic law" ([Neuzil and Tracy, 1981],[Wanfang et al., 1997],[Berkowitz, 2002]). One may also use a normalized longitudinal macroscopic permeability coefficient defined as :

$$
\frac{K_{x x}^{h o m}}{K_{x x, 0}^{h o m}}=\frac{\int_{X_{0}^{c \ell}(\Sigma)}^{\infty} X^{3} \epsilon\left(X_{0}\right) d X_{0}}{\int_{0}^{\infty} X_{0}^{3} \epsilon\left(X_{0}\right) d X_{0}}
$$

Clearly enough, permeability couplings with mechanical fracture reclosure is completely accounted for by both the initial crack aspect ratio distribution function $\epsilon\left(X_{0}\right)$ and the local crack reclosure law $X_{0}^{c \ell}(\Sigma)$. The originality of our contribution lies in the fact that both $\epsilon\left(X_{0}\right)$ and $X_{0}^{c \ell}(\Sigma)$ are exclusively identified from the mechanical fracture reclosure law measured experimentally.

\footnotetext{
${ }^{4}$ consistent with the Mori Tanaka scheme
} 


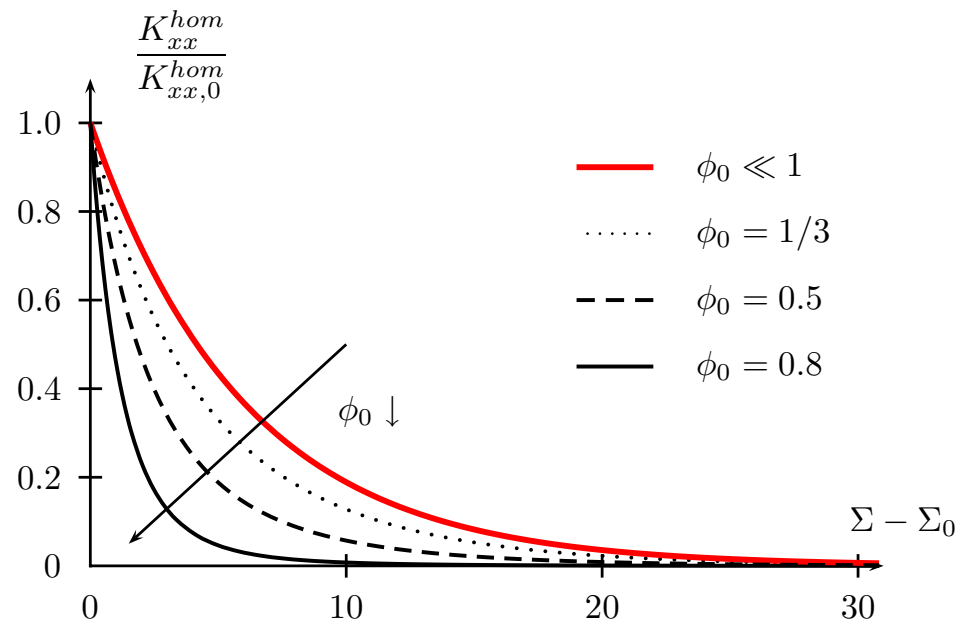

Figure 7: Effect of $\phi_{0}$ on the normalized permeability evolution, $\Sigma_{\infty}=6 \mathrm{MPa}$ )

\subsubsection{Effect of $\phi_{0}$ on the normalized permeability evolution}

As for the mechanical analysis developed in the previous section, the effect of $\phi_{0}$ may already be discussed. For $\alpha_{n}^{s}=5 e^{-5} \mathrm{MPa}^{-1}$ and $\Sigma_{\infty}=6 \mathrm{MPa}$, normalized permeability coefficient (40) is plotted vs confining pressure intensity for different values of $\phi_{0}$ in figure 7 . The following comments can be made:

- in the range $0 \leq \phi_{0} \leq 1 / 3$, theoretical estimates are very close to each other. Therefore, if theoretical estimates are close to the experimental results whatever $\left.\phi_{0} \in\right] 0,1 / 3$ ], it is possible to predict the hydraulic response of fractured materials directly from the mechanical fracture reclosure law identified experimentally. Indeed, in this case experimental results may be fairly well approximated by the estimate obtained in the limit case $\phi_{0} \rightarrow 0$ (Dilute scheme approximation [Lemarchand et al., 2007]) recalled in (49). The latter only requires the determination of parameter $\Sigma_{\infty}$.

- when $\phi_{0}>1 / 3$, there is no way to predict the hydraulic behavior from the mechanical one since $\phi_{0}$ is still unknown. By contrast, hydraulic behavior allows the determination of $\phi_{0}$ by best-fitting procedure.

\subsubsection{Effect of $\Sigma_{\infty}$ on the normalized permeability evolution}

Evolution of the normalized permeability coefficient with respect to the confining pressure is displayed in figure 8 for different values of parameter $\Sigma_{\infty}$ when $\phi_{0}=0.5$. Clearly enough, when $\Sigma_{\infty}$ decreases the permeability coefficient decreases more rapidly. This result is consistent with the aforementionned effect of $\Sigma_{\infty}$ on the initial aspect ratio distribution, where higher values of $\Sigma_{\infty}$ are associated with ill-mated fractures. 


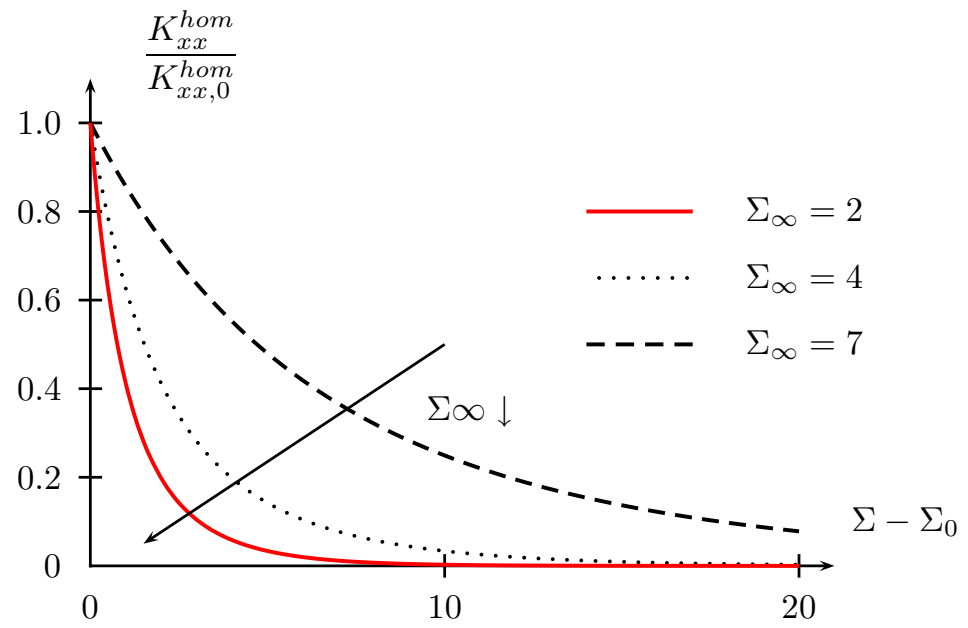

Figure 8: Effect of $\Sigma_{\infty}$ on the normalized permeability evolution, $\phi_{0}=0.5$ )

\section{Application}

\subsection{Experiments}

The experimental method is fully described in [Davy et al., 2007], as applied to Callovo-Oxfordian argillite, which is a deep geological formation privileged for deep underground nuclear waste storage. The results presented here are upon industrial high performance concretes which are studied by Andra (French Agency for Nuclear Waste Management) in similar context (i.e. deep nuclear waste storage), and also by EDF (French Electricity Supply Company) for nuclear reactor vessels. Both concretes, labelled CEM I and CEM V respectively, are made of 4-12 $\mathrm{mm}$ calcareous aggregates, of CEM I-type or alternately CEM V-type cement, of a water proportion on the order of $40 \%$ cement mass (i.e. water-to-cement ratio $\mathrm{W} / \mathrm{C} \approx 0.4)$ and of a superplasticizer, in proportions as requested by ANDRA. For theoretical purpose, concrete behavior is assumed isotropic elastic: Young's modulus $E_{s}=30 \mathrm{GPa}$ and Poisson's ratio $\nu_{s}=0.5$.

\begin{tabular}{|c|c|c|c|c|c|c|}
\hline \multirow{2}{*}{$\begin{array}{c}\text { Sample } \\
\text { number }\end{array}$} & \multicolumn{3}{|c|}{ Reference Configuration } & \multicolumn{3}{c|}{ Final Configuration } \\
\cline { 2 - 7 } & $\begin{array}{c}\Sigma_{0} \\
{[\mathrm{MPa}]}\end{array}$ & $\begin{array}{c}\Delta_{0}^{c} \\
{\left[10^{-5} \mathrm{~m}\right]}\end{array}$ & $\begin{array}{c}K_{x x, 0} \\
{\left[10^{-15} \mathrm{~m}^{2}\right]}\end{array}$ & $\begin{array}{c}\Sigma_{\max } \\
{[\mathrm{MPa}]}\end{array}$ & $\begin{array}{c}\Delta_{\max }^{c} \\
{\left[10^{-5} \mathrm{~m}\right]}\end{array}$ & $\begin{array}{c}K_{x x, \max } \\
{\left[10^{-15} \mathrm{~m}^{2}\right]}\end{array}$ \\
\hline Sample 1 & 1.8 & 0 & 32 & 43.2 & 3.91 & 1.3 \\
Sample 2 & 3.6 & 1.9 & 0.88 & 45.1 & 5.4 & 0.022 \\
\hline
\end{tabular}

Table 1: Gas permeability test results on fractured concrete samples subjected to hydrostatic pressure $\Sigma$. Gas pressure for permeability assessment is of $1 \mathrm{MPa}$ in both cases.

A Brazilian splitting test reproduces a fracture along the diametral plane of a circular cylindrical specimen, see Figure 1(b). All specimens have a length- 


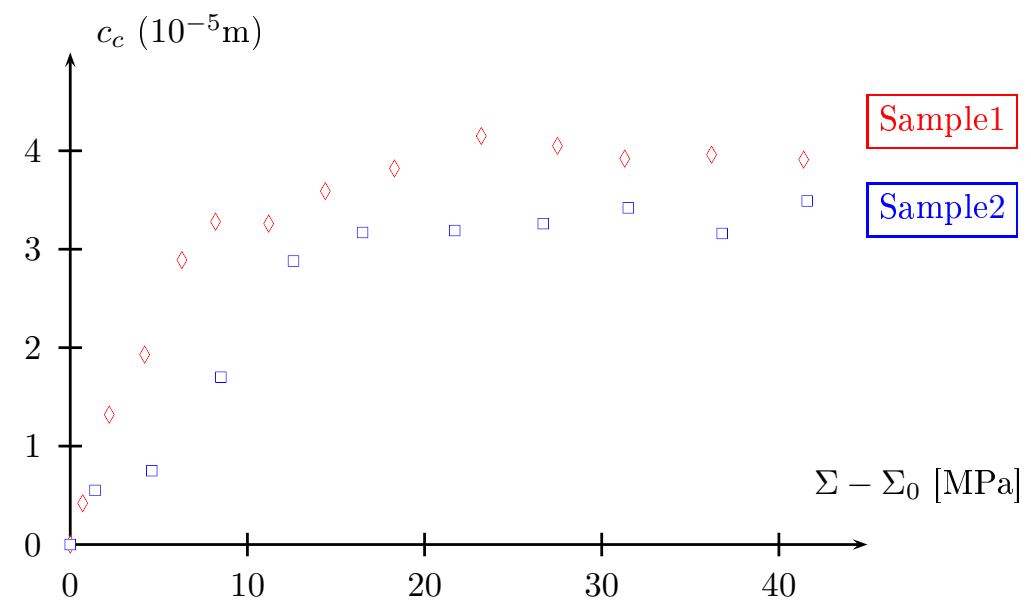

Figure 9: Experimental measurements of $c_{c}=\left(\Delta^{c}-\Delta_{0}^{c}\right)(\Sigma)$

to-diameter ratio $(L / D)$ approaching 2 (with $D \approx 37 \mathrm{~mm}$ and $L \approx 70 \mathrm{~mm}$ ). Although only two test results are presented here, several samples have been tested, so as to ensure proper results representativity. Sample 1 is made with CEM V cement $(\mathrm{W} / \mathrm{C}=0.39)$ and Sample 2 is composed of CEM I cement $(\mathrm{W} / \mathrm{C}=0.43)$. Subsequently, each sample is wrapped in a Vitton membrane. It is then subjected to an hydrostatic compressive loading in a triaxial cell with a maximum hydrostatic pressure $\Sigma$ of $45 \mathrm{MPa}$. The measured crack closure $\Delta^{c}$ is evaluated from the average displacement of four LVDT sensors placed diametrically around the sample. Preliminary calibration phase enables to account for all set-up and bulk concrete deformations, so that finally, $\Delta^{c}$ data correspond solely to fracture closure or opening. Evaluation of longitudinal gas permeability $K_{x x}$ is performed under quasi-static inert Argon gas flow, at pressures of 0.5 or $1 \mathrm{MPa}$, using Darcy's law and perfect gas assumption, see [Davy et al., 2007] for details. The permeability evaluated here is qualified as apparent, in the sense that gas slippage effects are not accounted for. The expression providing $K_{x x}$ remains identical for a fractured specimen as for an intact one, although gas flows preferentially through the fracture rather than through the material bulk. Indeed, volume flow rate is identical at any given cross-section along $\underline{e}_{x}$. $K_{x x}$ is not normalized by sample diameter $D$ so as to get a fracture permeability, we rather work with apparent fractured sample permeability (expressed in $\mathrm{m}^{2}$ ). Moreover, while $\Delta^{c}$ is measured instantaneously, permeability measurements $K_{x x}$ are conducted at stabilized values of confining pressure $\Sigma$. Initial (reference state) and asymptotic values are summarized in Table 1.

As shown in Figure 9, when applying hydrostatic pressure loading up to $45 \mathrm{MPa}$, a progressive crack closure of great amplitude (of 40 or $50 \mu \mathrm{m}$ ) occurs. Similar observations are made for gas permeability relationship with hydrostatic loading: a sharp decrease of the normalized permeability coefficient $K_{x x}^{\exp } / K_{x x, 0}^{\exp }$ is obtained for both samples, see Figure 10. Disparities in permeability and crack closure amplitude between samples may be accounted for by the variability gen- 


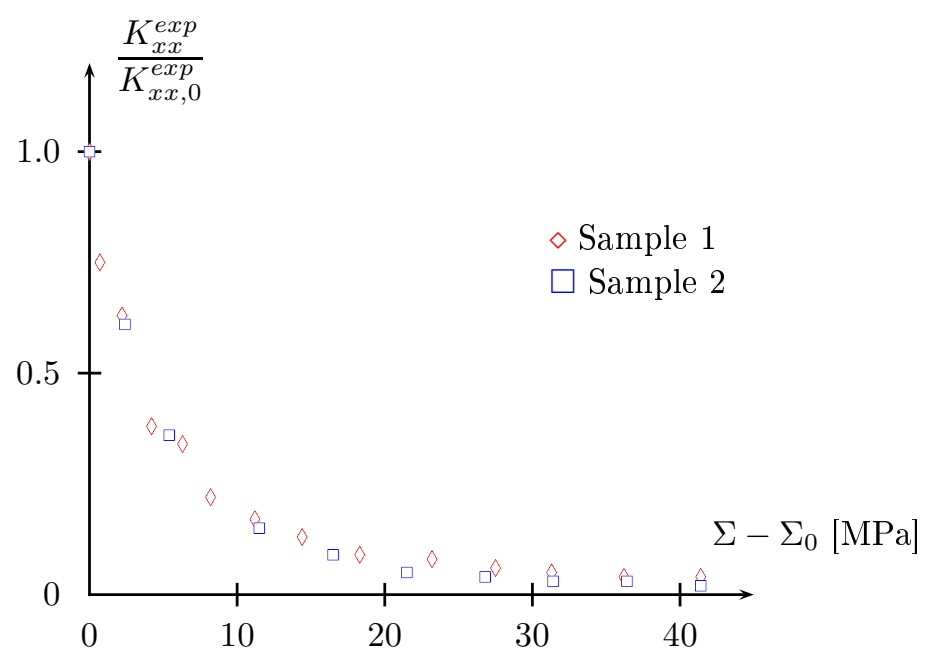

Figure 10: Normalized longitudinal permeability coefficient w.r.t. $\left(\Sigma-\Sigma_{0}\right)-$ experimental results

erated by the Brazilian splitting test. As stated above, this is yet in favour of a closer representation of the in situ crack profile variability.

\subsection{Theoretical results}

In this section, the micromechanics reasoning proposed in section 2.2 is applied and compared to experimental results obtained with fractured concrete samples.

\begin{tabular}{|c|cc|c|}
\hline Test $^{o}$ & $c_{c}^{\infty}$ & $\left(10^{-5} \mathrm{~m}\right)$ & $\Sigma_{\infty}(\mathrm{MPa})$ \\
\hline Sample 1 & 4 & 5.6 \\
\hline Sample 2 & 3.4 & 8.4 \\
\hline
\end{tabular}

Table 2: Identified parameters (least squares method)

According to section 2.2, the micromechanics methodology is directly related to the definition of the initial crack aspect ratio distribution $\epsilon\left(X_{0}\right)$ expressed in (30). This function, characterizing the morphology of the fracture interfacial domain, depends upon three unknown parameters: $c_{c}^{\infty}, \Sigma_{\infty}$ and $\phi_{0}$, which have to be identified. $c_{c}^{\infty}$ and $\Sigma_{\infty}$ are determined by taking advantage of the fracture reclosure law identified experimentally (see Fig. 9). In the range of applied confining pressures, the best fit between experimental and theoretical results (see Fig. 11) is provided by the least squares method. Black points are experimental measurements while the red solid line is the analytical expression given in (10) for the optimized values summarized in Table 2 for the two concrete samples. 

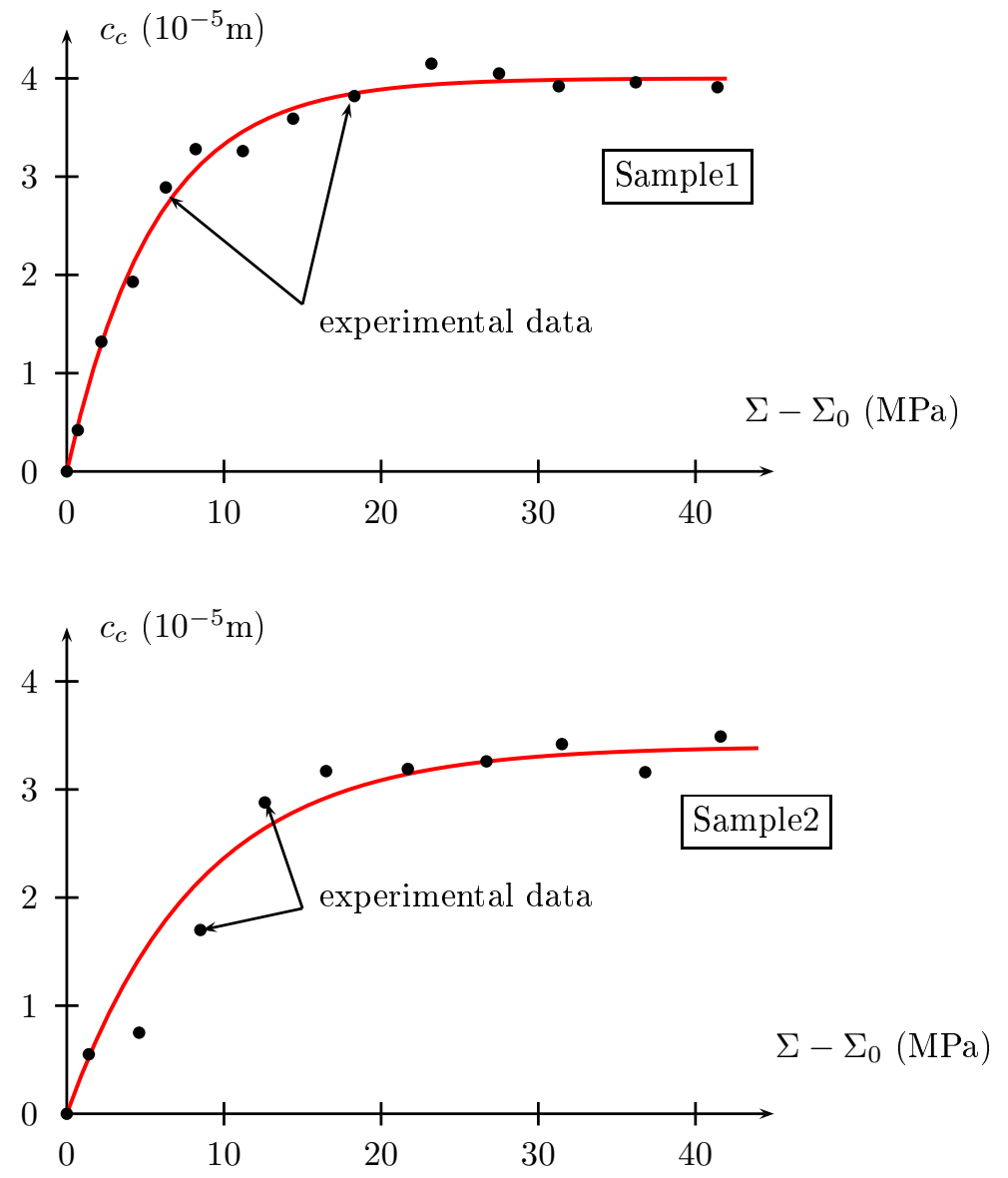

Figure 11: Experimental identification of $c_{c}=c_{c}(\Sigma)$ 
With these values in hand, we are able to define the initial crack aspect ratio distribution (30) and the crack closure law (25) as functions of the unknown initial fracture pore volume fraction $\phi_{0}$. The latter is sought in order to get the best fit between experimental and micromechanics results obtained for the normalized permeability coefficient evolution (40) as displayed in Figure 12. Obviously, micromechanics-based theoretical estimates are able to reproduce the experimental evolution of the normalized longitudinal permeability coefficient. More precisely, the Mori-Tanaka estimate (40) provided by our modelling fits experimental results, at least in the range $\Sigma: 0 \rightarrow 20 \mathrm{MPa}$, for $\phi_{0}=0.33$ (resp. $\phi_{0}=0.37$ ) for Sample 1 (resp. Sample 2).

Therefore, the mixture law (39), consistent with a local cubic law of the permeability evolution with respect to crack aspect ratio, is capable of accounting for the physical mechanism, revealed by experimental evidences, that controls both mechanical and hydraulic behaviors of the tested fractured concrete samples.

Besides, experimental results suggest that the macroscopic confining pressure leads to a decrease of the permeability almost perfectly correlated with mechanical fracture reclosure. More precisely, for the very first loading values $\Sigma-\Sigma_{0} \approx 10 \mathrm{MPa}$, the normalized permeability coefficient decreases from 1 to 0.19 (Fig. 10) while, at the same time, the normalized fracture closure $\left(c_{c}^{\infty}-c_{c}\right) / c_{c}^{\infty}$ decreases from 1 to 0.17 (Fig. 9 - Fig. 10). These experimental results are at the origin of the good agreement between experiments and theoretical estimates derived by the dilute approximation $\left(\phi_{0} \rightarrow 0\right)$. The latter case has already been solved in [Lemarchand et al., 2007] (see appendix). It yields an affine relationship between the normalized permeability and the normalized fracture reclosure of the form :

$$
\frac{K_{x x}^{h o m}}{K_{x x, 0}^{h o m}}=1-\frac{c_{c}}{c_{c}^{\infty}}
$$

This corresponds to a perfect correlation between mechanical and hydraulic responses, which has been observed for concrete samples.

In contrast with the good agreement obtained between experimental data and theoretical estimates for confinement intensity in the range $\Sigma=0-20 \mathrm{MPa}$, one may observe a non-negligible discrepancy for $\Sigma=20$ to $-40 \mathrm{MPa}$ (Sample 1). This is a direct consequence of our morphological assumption in the definition of the fracture pore volume. Indeed, in addition to stress-sensitive crack-like pores, pores that are stress-insensitive should also be considered within the fracture domain. Therefore, these "non-LCL" regions 5 ([Wanfang et al., 1997],[Oron and Berkowitz, 1998],[Berkowitz, are expected to contribute to the hydraulic conductivity in a non negligible way. In particular, accounting for these pores in our micromechanics approach would certainly improve the asymptotic hydraulic behavior estimates.

\footnotetext{
${ }^{5}$ non Local Cubic Law regions
} 

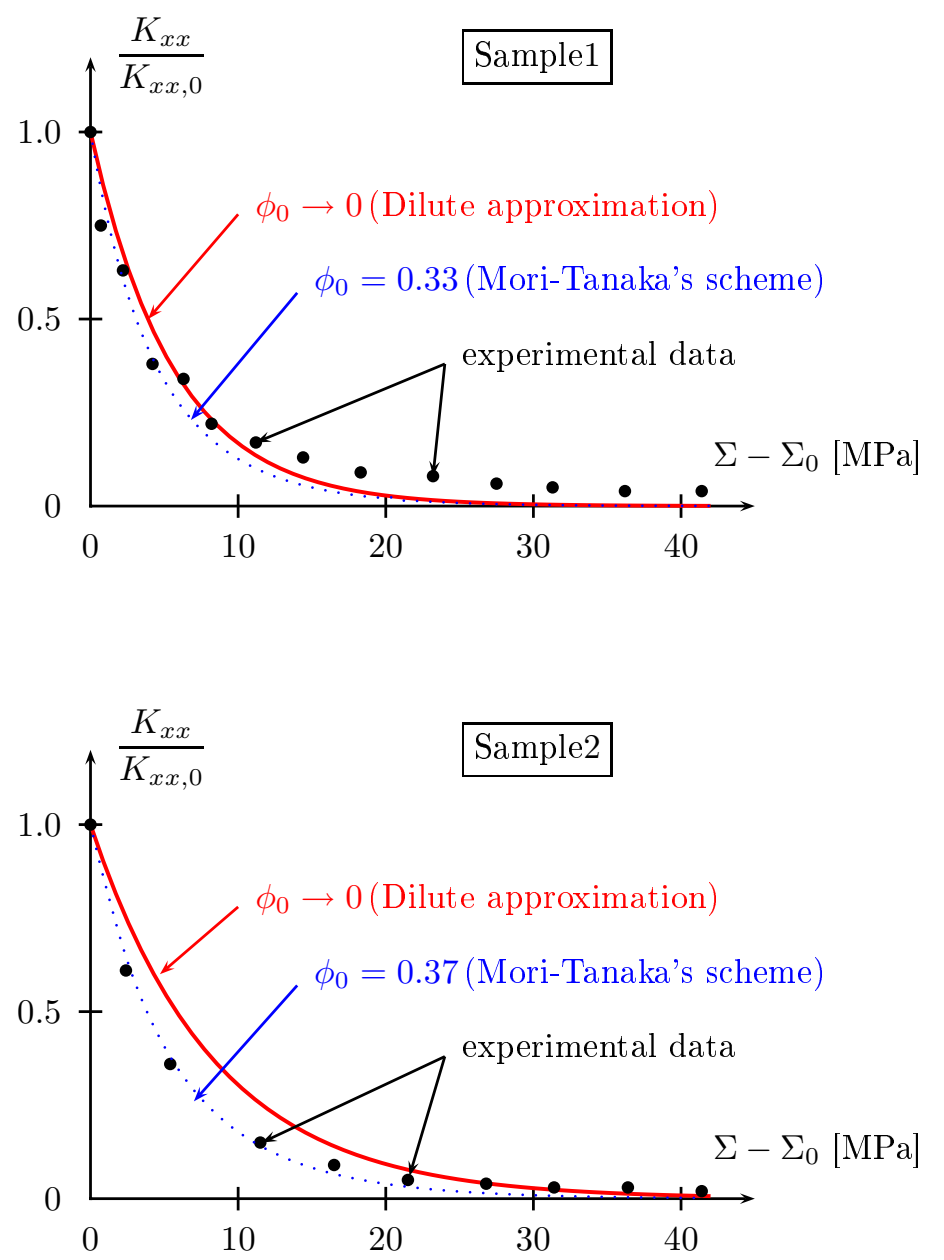

Figure 12: Normalized longitudinal permeability coefficient w.r.t. $\Sigma-\Sigma_{0}$ 


\section{Discussion - Conclusion}

In the present paper, we proposed a micromechanics modelling of fractured geomaterials accounting for interdependent hydraulic and mechanical behaviors. We adressed the question of a single through-wall fracture in a non conductive solid matrix. For compressive loadings, experimental results prove that both hydraulic and mechanical behaviors show a non linear evolution with respect to the confinement intensity. The observed non linearity of the fracture reclosure is physically interpreted as the progressive closure of crack-like pores defining the pore space within the fracture domain. This 3D interpretation of the macroscopic interface lies in the physical concept of roughness, which is usually introduced in order to account for fracture surface irregularities.

Disregarding tortuosity effects, we assumed a 2D definition of crack-like pores, represented by cylinders of elliptical section normal to the flow path. In the framework of Mori Tanaka effective media theory, we accounted for the possible interaction between these cracks for both mechanical and hydraulic behaviors. This micromechanics reasoning is controlled by two physical informations, a crack closure threshold $X_{0}^{c \ell}(\Sigma)$ and a distribution of initial aspect ratios $\epsilon\left(X_{0}\right)$ given in (25) and (30) respectively. To be completely determined, both need the experimental mechanical fracture reclosure law $\left(\Sigma_{\infty}\right)$ and the initial pore volume fraction of the fracture domain $\left(\phi_{0}\right)$. While the former is identified for the two tested concrete samples, the latter has not been measured experimentally, thus appearing in our model as an unknown parameter.

For concrete samples, best fits have been obtained with $\phi_{0}=0.33$ (Sample 1) and $\phi_{0}=0.37$ (Sample 2). Obviously, to be fully predictive, the proposed multi-scale modelling needs $\phi_{0}$, or equivalently $\lambda_{0}$ owing to (11), not to remain an unknown parameter. This goes through experimental characterization of the initial fracture pore volume, using imaging techniques for instance [Viggiani et al., 2004]. From these authors, X-ray microtomography observations made on fine-grained geomaterials proved that unconfined fracture thicknesses are in the order of 10-100 $\mu \mathrm{m}$. This characteristic size may be interpreted as that of the grains located on macro-crack lips which are responsible for the surface roughness. According to (11), its value is consistent with an initial fracture porosity $\phi_{0}$ ranging from 0.2 to 1 for the tested samples. Values of $\phi_{0} \approx 0.35$ that have been obtained by the micromechanics reasoning are therefore compatible with these microstructural observations. In a first approximation, theoretical results derived in the framework of the dilute approximation (no crack interaction) defined by $\phi_{0} \ll 1$ provide a fairly acceptable approximation of experimental data. This is interesting in the sense that the model is then predictive as regards the permeability evolution, in spite of the indetermination on $\phi_{0}$. According to our micromechanics modelling, this simplification is directly related to the obvious correlation between mechanical and hydraulic behaviors of the fractured concrete samples. This is related to negligible tortuosity effects upon the hydraulic response of the tested samples.

Many experimental observations ([Cook, 1992] for instance) show that flow through a fracture may decrease more rapidly. [Bernabe and Brace, 1982] proved 
that hydraulic behaviors are strongly influenced by the type of geomaterials considered. Even within the crystalline rocks familly, experimental data show a strong disparity. A possible explanation has been given by [Sisavath et al., 2003] through the study of creeping flow through a fracture of varying aperture by introducing sinusoidally-varying walls. Our point of view rather considers that tortuosity effects are responsible for the observed disparity of the hydraulic behavior of fractured geomaterials. Therefore, in order to discus the effect of local cracks connectedness that accounts for the existence of a tortuous flow path within the fracture domain, micromechanics requires a $3 \mathrm{D}$ morphological representation of these cracks [Montemagno and Pyrak-Nolte, 1995]. While this morphological assumption would have negligible impact on the mechanical response of fractured geomaterials, the decrease of hydraulic conductivities should be enhanced. In particular, the influence of the initial crack aspect ratio distribution should have a strong influence [Tsang, 1984]. In parallel, the question of hydraulic percolation ([Pyrak-Nolte et al., 1988],[Walsh et al., 1997]) should be adressed in order to explain decreases of several orders of magnitude along the progressive reclosure of a class of fractured geomaterials. All these open questions will be analysed in a further paper.

\section{Acknowledgments}

The authors are grateful to both EDF (French Electricity Supply Company) and Andra (French Agency for Nuclear Waste Management) for funding the experimental research program. 


\section{$5 \quad$ Appendix}

We recall hereafter the micromechanics definition of both mechanical and hydraulic behaviors of fractured geomaterials when assuming that the crack-like pores within the fracture domain do not interact [Lemarchand et al., 2007]. Concerning the local mechanism of cracks reclosure, classical results ([Neuzil and Tracy, 1981], [Myer, 2000], [Rutqvist and Stephansson, 2003]) are retrieved.

\section{1 the mechanical behavior}

Within the dilute approximation, the set of open cracks $\mathcal{O}(\Sigma)$ given by $(25)$ in the limit $\varphi \approx \phi_{0} \ll 1$ is controlled by the following crack closure law [Deudé et al., 2002]:

$$
X=X^{0}-X_{c \ell}^{0}(\Sigma) \quad \text { with } \quad X_{c \ell}^{0}(\Sigma)=\alpha_{n}^{s}\left(\Sigma-\Sigma_{0}\right)
$$

According to (42), the closure of a given crack family occurs as soon as the applied stress reaches the value $\Sigma^{c \ell}\left(X^{0}\right)=\Sigma_{0}+X^{0} / \alpha_{n}^{s}$ corresponding to $X=0$. Owing to (42), (14) may be rewritten as :

$$
\varphi=\int_{X_{c \ell}^{0}(\Sigma)}^{\infty} X \epsilon\left(X^{0}\right) d X^{0}
$$

From (43), we may give another definition of the crack density parameter $\epsilon\left(X^{0}\right)$. Indeed, a double derivative of (43) with respect to $\Sigma$ yields :

$$
\epsilon\left(X^{0}\right)=\frac{1}{\left(\alpha_{n}^{s}\right)^{2}} \frac{d^{2} \varphi}{d \Sigma^{2}} \mid \Sigma=\Sigma^{c \ell}\left(X^{0}\right)
$$

Eventually, taking advantage of (12) together with the assumption $J \approx 1$, (44) may be rewritten as $\left(\phi_{0} \ll 1\right)$ :

$$
\epsilon\left(X^{0}\right)=\frac{\phi_{0}}{\left(\alpha_{n}^{s} \Sigma_{\infty}\right)^{2}} \exp \left(-\frac{X^{0}}{\alpha_{n}^{s} \Sigma_{\infty}}\right)
$$

\section{2 the hydraulic permeability}

Taking the local crack closure law (45) into account, estimate for the longitudinal macroscopic permeability coefficient given by (39) may be rewritten as :

$$
K_{x x}^{h o m}=\frac{a^{2} \phi_{0}}{3\left(\alpha_{n}^{s} \Sigma_{\infty}\right)^{2}} \int_{X_{c \ell}(\Sigma)}^{\infty}\left(X^{0}-\alpha_{n}^{s}\left(\Sigma-\Sigma_{0}\right)\right)^{3} \exp \left(-\frac{X^{0}}{\alpha_{n}^{s} \Sigma_{\infty}}\right) d X^{0}
$$

The assumed continuous distribution of initial crack aspect ratios eventually allows us to obtain the monotonic decreasing function of $\Sigma$ :

$$
K_{x x}^{h o m}(\Sigma)=2 \phi_{0}\left(a \alpha_{n}^{s} \Sigma_{\infty}\right)^{2} \exp \left(\left(\Sigma_{0}-\Sigma\right) / \Sigma_{\infty}\right)=2\left(a \alpha_{n}^{s} \Sigma_{\infty}\right)^{2} \varphi(\Sigma)
$$

The initial longitudinal macroscopic permeability coefficient also reads :

$$
K_{x x, 0}^{h o m}=K_{x x}^{h o m}\left(\Sigma=\Sigma_{0}\right)=2 \phi_{0}\left(a \alpha_{n}^{s} \Sigma_{\infty}\right)^{2}
$$


so that the normalized longitudinal macroscopic permeability coefficient is strictly controlled by parameter $\Sigma_{\infty}$ according to :

$$
\frac{K_{x x}^{\text {hom }}}{K_{x x, 0}^{\text {hom }}}=\frac{\varphi(\Sigma)}{\phi_{0}}=\exp \left(\frac{\Sigma_{0}-\Sigma}{\Sigma_{\infty}}\right)
$$




\section{References}

[Berkowitz, 2002] Berkowitz, B. (2002). Characterizing flow and transport in fractured geological media: A review. Advances in Water Resources, 25:pp. 861-884.

[Bernabe and Brace, 1982] Bernabe, Y. and Brace, W. (1982). Permeability porosity pore geometry hot pressed calcite. Mechanics of Materials, 1(3):pp. $173-183$.

[Billaux et al., 1984] Billaux, D., Feuga, B., and Gentier, S. (1984). Etude théorique et en laboratoire du comportement d'une fracture rocheuse sous contrainte normale. Rev. Fr. Geotechnique, 26:pp. 21-29.

[Budiansky and O'Connell, 1976] Budiansky, B. and O'Connell, R. (1976). Elastic moduli of a cracked solid. Int. J. Solids Structures, 12:81-97.

[Cook, 1992] Cook, N. (1992). Natural joints in rock: mechanical, hydraulic and seismic behaviour and properties under normal stress. Int. J. of Rock Mech E Min. Sc. Geomech. Abs., 29:198-223.

[Davy et al., 2007] Davy, C., Skoczylas, F., Barnichon, J., and Lebon, P. (2007). Permeability of macro-cracked argilite under confinement: Gas and water testing. Physics and Chemistry of the Earth, 32:pp. 667-680.

[Deudé et al., 2002] Deudé, V., Dormieux, L., Kondo, D., and Maghous, S. (2002). Micromechanical approach to non-linear poroelasticity: Application to cracked rocks. J. Mech. Eng.

[Dormieux and Kondo, 2004] Dormieux, L. and Kondo, D. (2004). Approche micromécanique du couplage perméabilité-endommagement . Comptes Rendus Mécanique, 332(2):135-140.

[Dormieux et al., 2006] Dormieux, L., Kondo, D., and Ulm, F.-J. (2006). Microporomechanics. Publisher Wiley.

[Dormieux and Lemarchand, 2000] Dormieux, L. and Lemarchand, E. (2000). Modélisation macroscopique du transport diffusif: apport des méthodes de changement d'échelle d'espace. Oil \& Gas - Revue de l'Institut Francais du Pétrole, 55(1):15-34.

[Dormieux and Lemarchand, 2001] Dormieux, L. and Lemarchand, E. (2001). A homogenization approach of advection and diffusion in cracked porous material . Journal of Engineering Mechanics, 127(12):1267-1274.

[Eshelby, 1957] Eshelby, J. (1957). The determination of the elastic field of an ellipsoidal inclusion, and related problems. Proc. Roy. Soc. London, A241:235251. 
[Gentier, 1986] Gentier, S. (1986). Morphologie et comportement hydromécanique d'une fracture naturelle dans un granite sous contrainte normale. PhD thesis, Univ. d'Orléans, France.

[Gueguen and Palciauskas, 1992] Gueguen, Y. and Palciauskas, V. (1992). Introduction à la Physique des Roches. Publisher Hermann, Paris.

[Horii and Nemat-Nasser, 1983] Horii, H. and Nemat-Nasser, S. (1983). Overall moduli of solids with microcracks: load-induced anisotropy. J. Mech. Phys. Solids, 31(2):155-171.

[Hsieh et al., 1985] Hsieh, P., Neuman, S., Stiles, G., and Simpson, E. (1985). Field determination of three dimensional hydraulic conductivity tensor of anisotropic media, 2. methodology and application to fractured rock. Water Resources Research, 21:pp. 1667-1676.

[Iwai, 1976] Iwai, K. (1976). Fundamental studies of fluid flow through a single fracture. PhD thesis, University of California, Berkeley-CA.

[Jaeger et al., 2007] Jaeger, J., Cook, N., and Zimmerman, R. (2007). Fundamentals of Rock Mechanics - Fourth Edition, volume ISBN: 9780632057597, ISBN10: 0632057599. Blackwell Publishing.

[Lemarchand et al., 2007] Lemarchand, E., Dormieux, L., Davy, C. A., and Skoczylas, F. (2007). Analyse micromécanique du couplage perméabilitécontrainte d'une argilite fracturée. Revue Européenne de Génie Civil, 11(6):763-773.

[Montemagno and Pyrak-Nolte, 1995] Montemagno, C. and Pyrak-Nolte, L. (1995). Porosity of natural fracture networks. Geophysical Research Letters, 22(11(HS)):pp. 1397-1400.

[Moreno et al., 1988] Moreno, L., Tsang, Y., Tsang, C., and Neretnieks, I. (1988). Flow and tracer transport in a single fracture: a stochastic model and its relation to some field observations. Water Resources Research, 24:pp. 2033-2048.

[Morlier, 1971] Morlier, P. (1971). Description de l'état de fissuration d'une roche à partir d'essais simples et non-destructifs [Description of the state of rock fracturization through simple non-destructive tests]. Rock Mech., 3:125138.

[Mura, 1987] Mura, T. (1987). Micromechanics of defects in solids, 2nd Edition. Kluwer Academic Publishers.

[Myer, 2000] Myer, L. (2000). Fractures as collections of cracks. Int. J. Rock. Mech. and Min. Sci., 37:231-243.

[Neuzil and Tracy, 1981] Neuzil, C. and Tracy, J. (1981). Flow through fractures. Water Resources Research, 17(1):pp. 191-199. 
[Oron and Berkowitz, 1998] Oron, A. and Berkowitz, B. (1998). Flow in rock fractures: the local cubic law assumption reexamined. Water Resources Research, 34(11):pp. 2811-2825.

[Pyrak-Nolte et al., 1988] Pyrak-Nolte, L., Cook, N., and Nolte, D. (1988). Fluid percolation through single fractures. Geophysical Research Letters, 15(11):pp. 1247-1250.

[Pyrak-Nolte and Morris, 2000] Pyrak-Nolte, L. and Morris, J. (2000). Single fractures under norman stress: the relation between fracture specific stiffness and fluid flow. Int. J. Rock. Mech. and Min. Sci., 37:245-262.

[Renshaw, 1995] Renshaw, C. (1995). On the relationship between mechanical and hydraulic apertures in rough-walled fractures. J. Geophys. Res., 100(24):24629-24636.

[Rutqvist and Stephansson, 2003] Rutqvist, J. and Stephansson, O. (2003). The role of hydromechanical coupling in fractured rock engineering. $\mathrm{Hy}$ drogeology Journal, 11:7-40.

[Sausse, 2002] Sausse, J. (2002). Hydromechanical properties and alteration of natural fracture surfaces in the Soultz granite (Bas-Rhin, France). Tectonophysics, 348:169-185.

[Sisavath et al., 2003] Sisavath, S., Al-Yaaruby, A., Pain, C., and Zimmerman, R. (2003). A Simple Model for Deviations from the Cubic Law for a Fracture Undergoing Dilation or Closure. Pure and Applied Geophysics, 160(5/6):1009-1022.

[Snow, 1965] Snow, D. (1965). A parallel plate model of fractured permeable media. PhD thesis, University of California, Berkeley-CA.

[Tsang, 1984] Tsang, Y. (1984). The effect of tortuosity on fluid flow through a single fracture. Water Resources Research, 20(9):pp. 1209-1215.

[Tsang and Tsang, 1987] Tsang, Y. and Tsang, C. (1987). Channel model of flow through fractured media. Water Resources Research, 23(3):pp. 467-479.

[Tsang and Tsang, 1989] Tsang, Y. and Tsang, C. (1989). Flow chanelling single fracture as two dimensional strongly heterogeneous permeable medium. Water Resources Research, 25(9):pp. 2076-2080.

[Tsang and Witherspoon, 1983] Tsang, Y. and Witherspoon, P. (1983). The dependence of fracture mechanical and fluid flow properties on fracture roughness and sample size. Journal of Geophysical Research, 88(B3):pp. 2359-2366.

[Unger and Mase, 1993] Unger, A. and Mase, C. (1993). Numerical studiy of the hydromechanical behavior of two rough fracture surfaces in contact. Water Resour. Res, 29:2101-2114. 
[Viggiani et al., 2004] Viggiani et al., G. (2004). X-ray microtomography for studying localized deformation in fine-grained geomaterials under triaxial compresson. C.R. Méanique, 332:819-826.

[Walsh et al., 1997] Walsh, J., Brown, S., and Durham, W. (1997). Effective media theory with spatial correlation for flow in fracture. Journal of Geophysical Research, 102(B10):pp. 22587-22594.

[Walters and Wong, 1999] Walters, D. and Wong, R. (1999). The hydraulic and mechanical response of an oil sand fracture under varying confining pressure. Canadian Geotechnical Journal, 36(2):262-271.

[Wanfang et al., 1997] Wanfang, Z., Wheater, H., and Johnston, P. (1997). State of the art of modelling two-phase flow in fractured rock. Environmental Geology, 31(3/4):pp. 157-166.

[Witherspoon et al., 1980] Witherspoon, P., Wang, J., Iwai, K., and Gale, J. (1980). Validity of cubic law for fluid flow in a deformable rock fracture. Water Resources Research, 16(6):pp. 1016-1024.

[Zhou and Wheater, 1995] Zhou, W. and Wheater, H. (1995). Effect of aperture variation on two-phase flow in fractures., pages 173-182. IAHS Publication $\mathrm{N}^{\circ} 250$.

[Zimmerman and Bodvarsson, 1996] Zimmerman, R. and Bodvarsson, G. (1996). Hydraulic conductivity of rock fractures. Transport in Porous Media, $23: 1-30$ 